\title{
PID Controller Parameter Tuning Based on Improved Particle Swarm Optimization Algorithm
}

\author{
Yanzi Miao*, Yang Liu, Ying Chen and Huijie Jin \\ SIEE, China University of Mining and Technology, Xuzhou, China \\ ${ }^{*}$ Corresponding author
}

\begin{abstract}
Improved PSO algorithms combined with Simulated Annealing are proposed in this paper to solve the problems of the original PSO algorithm. After that three further improvements are proposed respectively from the parameters adjustment, the organization structure and evolution, and the topology structure. Four common test functions are used to test the optimizing performances of the improved PSO algorithms in the paper. The test results indicate that all the improved PSO algorithms have better performances compared with former PSO algorithms. Finally, the improved PSO algorithm is applied to PID parameter tuning, and the simulation experiment results prove that the improved PSO algorithm proposed in this paper is more feasible than other intelligent algorithms.
\end{abstract}

Keywords-particle swarm optimization (PSO); simulated annealing algorithm (SA); PID parameter tuning

\section{INTRODUCTION}

PID parameter tuning is the key technology of designing a control system, which is used to determine the parameter size of a PID controller according to different characteristics of every controlled object.

Particle Swarm Optimization (PSO) is a kind of parallel global search method based on population behavior, its ideal roots in artificial life and evolutionary computation theory. It operates according to the value of each individual size, and completes the process of optimizing. According to the fitness value of each individual, the best value of each particle and the best value of global population are found to complete the optimization of a process. Because of its simple concept, few parameter, and easy realization, it has attracted more and more attentions in recent years. At present, PSO has been used widely in parameter optimization and neural network training, fuzzy control system, system identification etc. [1, 2]. But the traditional Particle swarm algorithm easily sinks into the local optimal solution and produces precocious phenomena. For resolving the deficiencies of traditional PSO algorithm, a lot of works have been done by many scholars to improve the algorithm. One study [3] incorporated the Supervisor-Student model in PSO. One study [4] proposed a particle swarm algorithm combined with the mutation operation method, and the particle swarm intelligence factor was added according to the actual problem constraints. One study [5] presented the basic concepts of Particle Swarm Optimization (PSO) and its successful application in the different areas of water resources optimization. Self-adapting inertia can obtain better Convergent effect with the change of Iterative algebra was proved in the study [6]. In this paper, an improved particle swarm algorithm is proposed for the problems of PID controller parameter tuning, the simulation results prove that this method is more feasible and more reasonable than other intelligent methods.

\section{THE IMPROVED PSO ALGORITHM}

\section{A. Deficiency of the PSO Algorithm}

According to the optimization idea of PSO algorithm [7, 8], the solutions of every optimization problem are positions of particles in D-dimensional target search space, then $\mathrm{m}$ particles compose a swarm. The performance degree of every particle depends on the object functions fitness of pre-optimization problems, the fly direction and rate constitute the velocity of every particle, other particles search and follow the most optimal particle in solution space, so initializing PSO as swarm random particles to find the optimum solution with iterative method. Supposing a swarm of $\mathrm{N}$ particles search in Ddimension space, the positions of $\mathrm{N}$ particles which represent the solutions constitute a population described as $\mathrm{Xi}$, which is substituted in object function, then the adaptive value Fitness is gotten, and it weighs $\mathrm{Xi}$. Meanwhile, every particle has its fly velocity, which is described as Vi The best individual position of the ith particle during the fly is the best history position, which is described as $\mathrm{Pi}$, called pbest $(\mathrm{i})$, the corresponding individual fitness is the best fitness value, Fitness(i). As the best position of all particles is the global best place, which is described as Pg, called gbest, the corresponding fitness is the best global fitness value Fg. After $\mathrm{n}$ times iteration, the velocity vector for the calculation of the particle position in $\mathrm{j}$-dimension $(1 \leq j \leq D)$ in the next iteration is calculated as [8]:

$$
\begin{aligned}
v_{i j}(n+1)= & v_{i j}(n) w_{i}+\operatorname{rand}_{1} c_{1}\left[\text { pbest }_{i j}-x_{i j}(n)\right] \\
& +\operatorname{rand}_{2} c_{2}\left[\text { gbest }_{j}-x_{i j}(n)\right]
\end{aligned}
$$

where, $\mathrm{n}$ is the iteration time, rand1,rand 2 is random number between 0 and 1, c1, c2 is learning factor, wi is inertia weight. If the velocity of particle exceeds edges during iteration process, then the boundary value is chosen.

\section{B. The Improved Particle Swarm Algorithm}

First, we handle the initial population in chaos initialization. Generating $\mathrm{n}$ random numbers in interval $(0,1)$, Chaos operation mark counter $n=0$. Chaos generated the initial particle, and the iteration number of Logistic is described as follows:

$$
z(t+1)=\mu^{*} z(t) *(1-z(t))
$$


Then we calculate the fitness function value of each particle in each iteration, and select $\mathrm{m}$ particles which have the optimal fitness as the initial particle.

According to the Metropolis rule [9], all the particles from the chaotic interval $(0,1)$ are mapped to the variable value range.

Particle swarm algorithm easily sinks into the local optimal solution and diversity of particles disappears. For resolving these deficiencies, this paper introduces an improved particle swarm optimization algorithm amalgamated with a chaos simulated annealing algorithm.

In the chaos simulated annealing particle swarm optimization algorithm, we hope that every generation of algorithm operation can generate at least one new status for each particle, and accept the new status of the poor in a certain probability. This means that the particles may move into the other location which is worse than current location. If the current location is the local optimum, so the particle jumped out of the local optimum. The probability that the particles accept the new status controlled by the temperature, the lower the temperature is, the lower the probability is. This design idea can improve the global search ability in the previous algorithm (high temperature), and ensure overall convergence in the advanced algorithm (low temperature). In the following, we discuss some key parameters and operation design of the annealing POS.

The new state produces function: In the simulated annealing algorithm, the Starting point that the new state produces function should ensure that the best possible candidate of the solution can cover the full solution space. Usually, the state function is composed of two parts: the way of generating candidate solutions and the probability distribution of the candidate solutions. The former decides the way in which current solution produces candidate solutions. The later decides the probability of choosing different states in candidate solutions produced by current solution. The way of generating candidate solutions decided by the nature of the problem, it usually generates at a certain probability in the neighborhood structures of present status, and neighborhood function and probability can be diversified designed.

In the chaos simulated annealing particle swarm optimization algorithm, we increase random disturbance for current solution, choose this way as the Particles new location produce function. Supposing $x(i, j)$ is the position coordinates of random particle from particle swarm in $\mathrm{j}$-dimension, this paper chooses following new location produce function:

$$
x(i, j)=x(i, j) *(-4+2 * \text { rand })
$$

In which, rand belongs to uniform distribution of $[0,1]$.

\section{Test on Algorithms Performances}

The Sphere and Rastrigrin Functions[10] are adopted as testing function for comparing the original PSO algorithm, simulated annealing algorithm, with our improved PSO algorithm, which amalgamated with improved chaos simulated annealing algorithm.
The formula of Sphere Function is described as follows:

$$
f_{1}(x)=\sum_{i=1}^{n} x_{i}^{2},-100 \leq x_{i} \leq 100
$$

It is a function with only global extreme point and without local extreme point. The global extreme point is the origin, and its function value is 0 , which is easy to find out.

The Rastrigrin Function is a function with only global extreme point and with multiple local extreme points, and the global extreme point is surrounded by more local extreme point. The global minimum of this function is in the line of $x i=0$, the minimum value of function is 0 . Generally, it is difficult to find out the global minimum point of the Rastrigrin Function. The formula of Rastrigrin Function is described as:

$$
f_{2}(x)=\sum_{i=1}^{n}\left[x_{i}^{2}-10 \cos \left(2 \pi x_{i}\right)+10\right],-5.12 \leq x_{i} \leq 5.12
$$

In this test experiments, the population of simulation particles is 20 , the iteration time from terminate rule is given as 1000 times. The simulation experiments of three algorithms run 24 times respectively. For the results of simulation, both the two highest and two lowest solutions are removed, then the average value of the rest is gotten. Supposing the dimension is 30 , the results of fitness with Sphere Function and Rastrigrin Function in 30-dimension are calculated respectively, as shown in Table I and Table II, and the evolving currents of the best fitness with each function are recorded respectively from Figure I to Figure II.

TABLE I. THE RESULTS OF FITNESS WITH THE SPHERE FUNCTION IN 30-DIMENSION

\begin{tabular}{cccc}
\hline Algorithm & PSO & SA PSO & $\begin{array}{c}\text { Improved } \\
\text { PSO }\end{array}$ \\
\hline Minimum value & $1.026355 \mathrm{e}-002$ & $4.852753 \mathrm{e}-009$ & $2.393488 \mathrm{e}-020$ \\
Maximum value & $1.970301 \mathrm{e}-001$ & $3.455452 \mathrm{e}-004$ & $3.178690 \mathrm{e}-016$ \\
Average value & $5.532385 \mathrm{e}-002$ & $6.382574 \mathrm{e}-005$ & $2.323788 \mathrm{e}-018$ \\
\hline
\end{tabular}

TABLE II. THE RESULTS OF FITNESS WITH THE RASTRIGRIN FUNCTION IN 30-DIMENSION

\begin{tabular}{cccc}
\hline Algorithm & PSO & SA PSO & $\begin{array}{c}\text { Improved } \\
\text { PSO }\end{array}$ \\
\hline Minimum value & $1.168209 \mathrm{e}+002$ & $2.344901 \mathrm{e}-002$ & $3.552714 \mathrm{e}-015$ \\
Maximum value & $2.948199 \mathrm{e}+002$ & $1.147815 \mathrm{e}+002$ & $3.328908 \mathrm{e}-008$ \\
Average value & $2.088337 \mathrm{e}+002$ & $3.442423 \mathrm{e}+001$ & $3.106795 \mathrm{e}-011$ \\
\hline
\end{tabular}




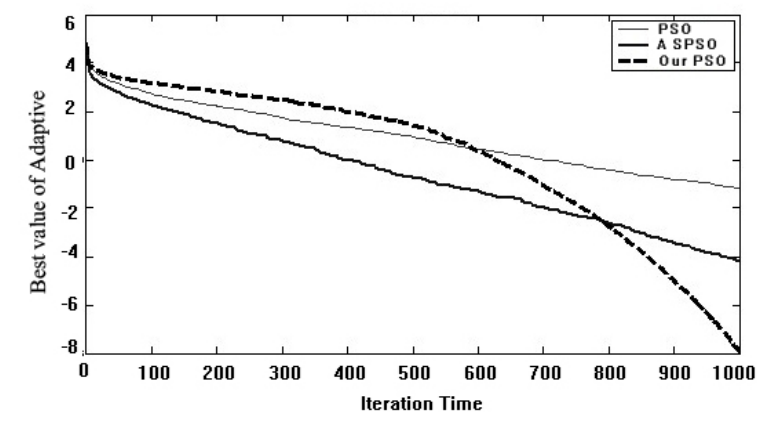

FIGURE I. EVOLVING CURRENTS OF THE BEST FITNESS WITH THE SPHERE FUNCTION IN 30-DIMENSION

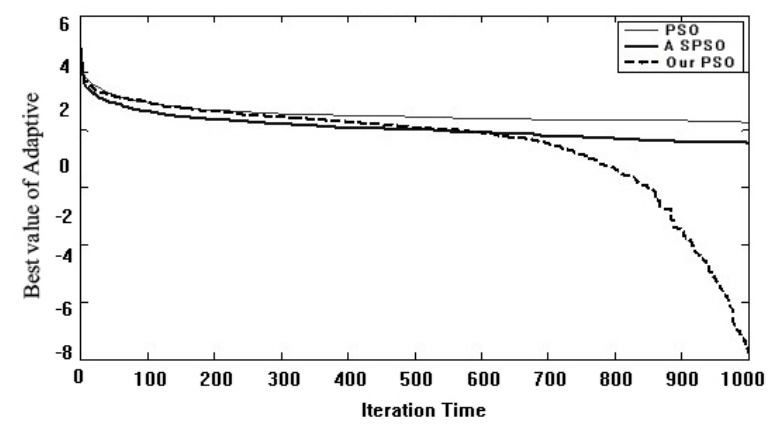

FIGURE II. EVOLVING CURRENTS OF THE BEST FITNESS WITH THE RASTRIGRIN FUNCTION IN 30-DIMENSION

\section{PID PARAMETER TUNING BASED ON IMPROVED PSO ALGORITHM}

PID controller occupies important position in engineering control, which is applied in more than $90 \%$ circuit, as it is the basic of many advanced control [11]. The traditional common methods of PID parameter tuning are orthogonal testing method, stochastic optimization method, the pole assignment method, Nelder-Mead method, Latin-Hypercube Sampling, and so on, they have each advantages but also obvious flaws. For example, orthogonal testing method has strong dependency on initial parameters, high testing error and low optimum efficiency. Traditional stochastic optimization method has its own technical limitations and low efficiency. The convergence and optimum efficiency of the pole assignment method are influenced by structure model, constraint conditions and initial value of parameters.

The steps of optimizing PID parameters with improved PSO algorithm are described as:

(1) According to the experience, the range of PID controller parameter $\mathrm{Kp}, \mathrm{Ki}, \mathrm{Kd}$ and the control parameter of PSO algorithm Vmax is measured, then a swarm of particles in parameters range is initialized, that is, randomly generated position and speed.

(2) The fitness of every particle Ji is determined; using the unit step response ITAE is gotten with simulation computation, and then the fitness $\mathrm{Ji}$ is calculated.
(3) To update the best position of individual of every particle, and the global optimum position.

(4) To update the velocity and position of every particle, in which, $\mathrm{x} 1, \mathrm{x} 2$ and $\mathrm{x} 3$ stands for three parameters positions of PID controller, $\mathrm{Kp}, \mathrm{Ki}, \mathrm{Kd}$.

(5) If it is not satisfied the end condition (as presupposed algebra), then return to step (2).

\section{SIMULATION EXPERIMENTS}

For validating the effects of our improved PSO algorithm, the simulation experiments have been done to compare the improved PSO algorithm with other intelligent algorithms, the structure of simulation system is shown in Figure III. The controlled plant model is gotten from a motor servo system and its transfer function is described as:

$$
G_{\mathrm{p}}(\mathrm{s})=\frac{400}{\mathrm{~s}^{2}+50 \mathrm{~s}}
$$

From the experiments, the simulation results of PID controller parameter tuning with our improved PSO algorithm are compared to other common parameter tuning methods, Nelder-Mead and GA, and the step response of simulation system are acquired respectively and shown in Figure V. Three consecutive testing results are acquired using the NCD toolbox in Matlab software, the parameter setting comparation and the tunned parameters of PID controller from Nelder-Mead,, GA, and the improved PSO algorithm respectively are shown in Table III and Table IV as follows:

TABLE III. THE SETTING PARAMETERS OF ALGORITHMS

\begin{tabular}{cccc}
\hline Parameter setting & Nelder-Mead & GA & Improved PSO \\
\hline Particle number $(\mathrm{m})$ & & & 10 \\
Iteration time MAXITER & 20 & 20 & 20 \\
Frequencyof sampling & & & 5 \\
\hline
\end{tabular}

TABLE IV. THE PID PARAMETERS OF ALGORITHMS

\begin{tabular}{cccc}
\hline Algorithm & Kp & Ki & Kd \\
\hline Nelder-Mead & 3.71 & 0.52 & 1.32 \\
GA & 1.12 & 4.24 & 0.92 \\
Improved PSO & 3.82 & 0.37 & 0.53 \\
\hline
\end{tabular}

From the results above, combing with the chaos annealing particle swarm, the result of the improved PSO algorithm has the best optimization effect, and the Nelder-Mead had the worst effect. During the test, the Nelder-Mead method strongly depends on the initial value, GA is also affected in weak degree, but the improved PSO algorithm could not be influenced basically, it has excellent performance. With the improved PSO algorithm, the PID controller parameter tuning could get more flexible and more reasonable results than other common methods. 


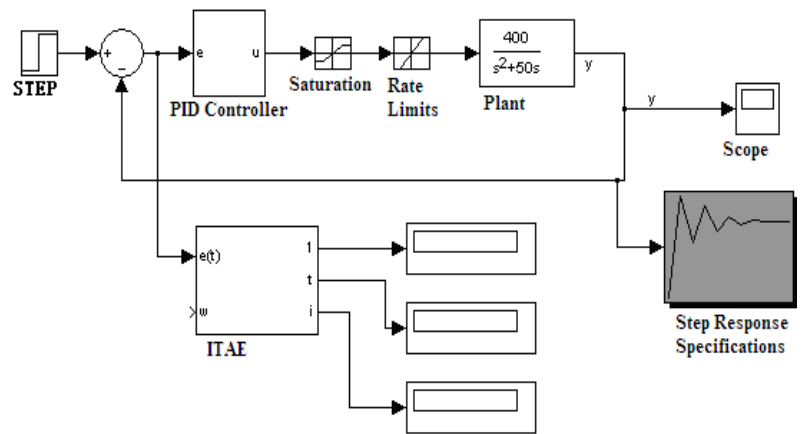

FIGURE III. SIMULATION OF PARAMETER TUNING

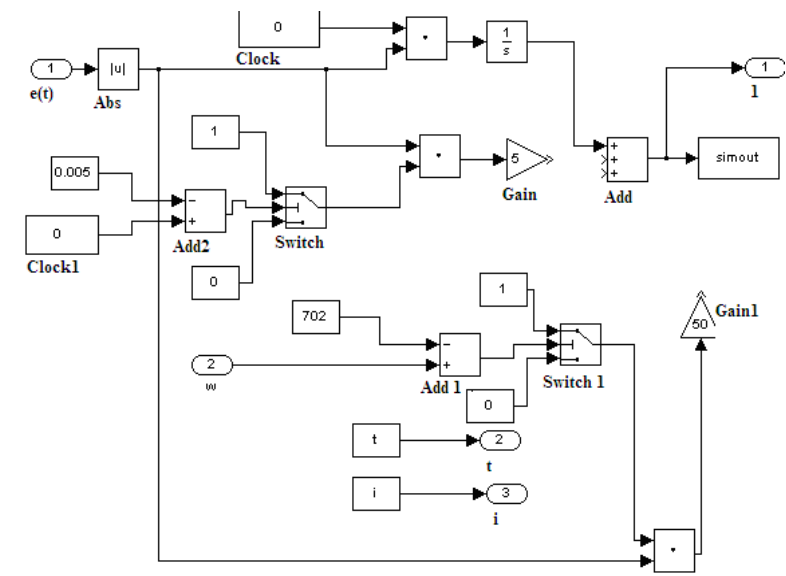

FIGURE IV. SIMULINK OF ITAE PARAMETER REGULATE FUNCTION

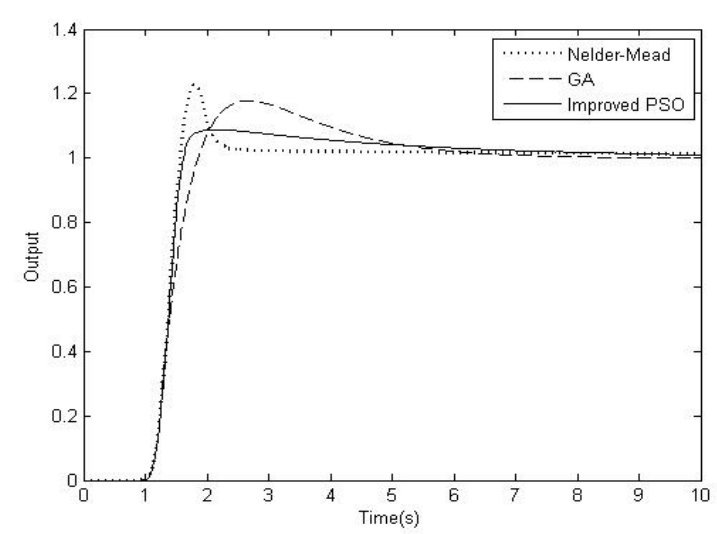

FIGURE V.

\section{SIMULATION RESULTS WITH DIFFERENT METHODS}

\section{CONCLUSIONS}

On the basis of analyzing the particle speed and position evolution equation of the original PSO algorithm, a kind of new improved PSO algorithm amalgamated with chaos simulated annealing algorithm is proposed and studied in this paper, and its application on designing the PID controller parameter tuning and optimized the three parameters of the PID controller are also described. The comparisons of the best fitness evolving currents with the Sphere and Rastrigrin Functions in 30-dimension indicate that the improved PSO algorithm described in this paper has better performance than original PSO, normal simulated annealing PSO. The simulation results of PID parameter tuning show that the controller gone through parameter optimization by the improved PSO algorithm can get much better control effect than that designed by other common intelligent method.

\section{ACKNOWLEDGMENT}

This work was supported by the National Natural Science Foundation of China (NO.61303183), the Natural Science Foundation of Jiangsu Province (NO.BK20130204), and the Specialized Research Fund for the Doctoral Program of High Education (NO.20120095120023).

\section{REFERENCES}

[1] S.Ch, S.Mathur, "Particle swarm optimization trained neural network for aquifer parameter estimation,’KSCE Journal of Civil Engineering, Vol. 16, pp. 298-307, 2012.

[2] Z.H. Zhan, J. Zhang, Y.Li, H.S.-H, "Adaptive particle swarm optimization". IEEE Transactions On Systems, Man, And Cyberneticspart B: Cybernetics, pp. 1083-4419, 2009.

[3] G.C.Chen, J.S.Yu, "Enhanced Particle Swarm Algorithm and its Application on Soft Measurement," Control and Decision, Vol.4, pp. 377-381, 2005

[4] P.X.Ding, S.R. Zou, H.W.Zhang, "Improved Particle Swarm Algorithm and Its Application in Vehicle Routing Problem," Advances in Intelligent and Soft Computing, Software Engineering and Knowledge Engineering: Theory and Practice. Vol.114, pp. 269-274, 2012.

[5] R.Cyriac, A.K.Rastogi, "An Overview of the Applications ofParticle Swarm in Water Resources Optimization," Advances in Intelligent Systems and Computing, Proceedings of Seventh International Conference on Bio-Inspired Computing: Theories and Applications (BIC-TA 2012), Vol, 202, pp. 41-52, 2013.

[6] Xie X F, Zhang W J, Yang Z L, "Overview of Particle SwarmAlgorithm," Control and Decision, Vol. 18, pp. 129-134, 2003.

[7] F.S.Wang,L.H.Chen, "Particle Swarm Optimization (PSO)," Encyclopedia of Systems Biology, Springer New York, pp.1649-1650, 2013.

[8] F.Wang, Research on Particle Swarm Algorithm, Southwest university press, Chengdu, 2006.

[9] A.Nickabadi, M. M. Ebadzadeh, R. Safabakhsh, "A competitiveclustering particle swarm optimizer for dynamic optimization problems," Swarm Intell, Vol.6, pp. 177-206, 2012.

[10] C.H.Dou, J.S.Lin, "Improved Particle Swarm Optimization Based on Genetic Algorithm," Advances in Intelligent and Soft Computing, Software Engineering and Knowledge Engineering: Theory and Practice, Vol.115, pp.149-153, 2012.

[11] X.D.Chen, Y.M.Zhang, "Optimum Design of PID Controller Parameters by Improved Particle Swarm Optimization Algorithm," Lecture Notes in Electrical Engineering, Recent Advances in Computer Science and Information Engineering, Vol.125, pp.79-84, 2012. 